\title{
A Study on Pharmacokinetics of Bosentan with Systems Modeling, Part 2: Prospectively Predicting Systemic and Liver Exposure in Healthy Subjects ${ }^{\text {『 }}$
}

\author{
Rui Li, Emi Kimoto, Mark Niosi, David A. Tess, Jian Lin, Larry M. Tremaine, and Li Di \\ Systems Modeling and Simulation, Medicine Design, Pfizer Worldwide R\&D, Cambridge, Massachusetts (R.L.); and \\ Pharmacokinetics, Dynamics, and Metabolism, Medicine Design, Pfizer Worldwide R\&D, Groton, Connecticut (E.K., M.N., \\ D.A.T., J.L., L.M.T., L.D.)
}

Received September 29, 2017; accepted January 8, 2018

\begin{abstract}
Predicting human pharmacokinetics of novel compounds is a critical step in drug discovery and clinical study design but continues to be a challenging task for hepatic transporter substrates, particularly in predicting their liver exposures. In this study, using bosentan as an example, we prospectively predicted systemic exposure and the (pseudo) steady-state unbound liver-to-unbound plasma ratio $\left(K \mathrm{puu}_{\mathrm{uu}}\right)$ in healthy subjects using 1) a mechanistic approach solely based on in vitro hepatocyte assays and 2) an approach based on hepatic
\end{abstract}

\section{Introduction}

Predicting human pharmacokinetic (PK) profiles of novel drug candidates is critical in drug discovery and development to project dose, determine the therapeutic index, and estimate drug-drug interaction (DDI) potential. With improved understanding of hepatic cytochrome P450 (P450) activity, relatively confident approaches have been developed in predicting hepatic P450-mediated small molecule clearance. These approaches have enabled the chemistry design to reduce P450-mediated metabolism for a prolonged drug half-life. However, such efforts have also led to an increased prevalence of hepatic transporter-mediated clearance, for which understanding is still limited as of today. As a result, for hepatic transporter substrates, even with reasonable estimates of hepatic P450 activity and volume of distribution, the systemic exposure may be mispredicted due to a failure in predicting liver exposure elevated or lowered by transporters. In addition, the ability to accurately predict liver exposure is also a foundation for predicting pharmacodynamics, DDI, and drug-induced toxicity within the liver.

Most approaches developed to predict exposures of transporter substrates are based on in vitro assays. Unfortunately, most in vitro transport rates cannot describe in vivo data without empirical scaling factors (i.e., fudge factors closing the gap between physiologic prediction and clinical observation), whether with a physiologically based pharmacokinetic (PBPK) model or static PK equations (Li et al., 2014a). The empirical scaling factors are the primary limitations associated with approaches based on in vitro assays, because most published values are

doi.org/10.1124/dmd.117.078808.

S This article has supplemental material available at dmd.aspetjournals.org. process rates from monkey in vivo data but Michaelis-Menten constants from in vitro data. Both methods reasonably match the observed human systemic time course data, but the second method leads to better prediction accuracy. In addition, the second method can predict a human $K p_{u u}$ value that is close to the value deduced using clinical data. We also generated rat and monkey liver $K \mathbf{p}_{\mathrm{uu}}$ values in terminal studies. However, these directly measured animal values are different from the deduced human value.

ABBREVIATIONS: DDI, drug-drug interaction; ET, endothelin receptor; GSK269984A, sodium; 6-[[5-chloro-2-[(4-chloro-2-fluorophenyl)methoxy]phenyl]methyl]pyridine-2-carboxylate, ; IVIVE, in vitro to in vivo extrapolation; MCMC, Markov chain Monte Carlo; OATP, organic anion-transporting polypeptide; P450, cytochrome P450; PBPK, physiologically based pharmacokinetic; PET, positron emission tomography; PK, pharmacokinetics; $\mathrm{PMH}$, plated monkey hepatocyte; RSV, rifamycin SV; $\mathrm{SCHH}$, sandwich cultured human hepatocyte. 
its liver exposure through analysis on systemic exposure and 2) develop an approach to prospectively predict its liver exposure. In part 1 of this work (Li et al., 2018), human liver exposure of bosentan was estimated from its systemic exposure using a "deduction" method. With the deduced bosentan liver exposure, we can compare the accuracy of both established and novel approaches to predict systemic and liver exposure described here (part 2).

\section{Materials and Methods}

\section{Human and Monkey Hepatocyte Uptake Assay and a Mechanistic In Vitro} Model

To understand the nonlinear transporter activity and intracellular binding processes, both sandwich cultured human hepatocyte $(\mathrm{SCHH})$ and plated monkey hepatocyte $(\mathrm{PMH})$ assays were performed with multiple dosing concentrations. The SCHH assay (lot HH1026, female donor; In Vitro ADMET, Columbia, MD) was carried out as described previously ( $\mathrm{Li}$ et al., 2014c), except that $1 \mathrm{mM}$ rifamycin SV (RSV) was used as transporter inhibitor. The method for the PMH assay (lot 10106012; In Vitro ADMET) is provided in the Supplemental Material.

A mechanistic model was developed to analyze the data (eqs. 1-4). The model includes concentrations of unbound extracellular $\left(C_{\mathrm{HEP}, \mathrm{UEC}}\right)$, unbound intracellular $\left(C_{\mathrm{HEP}, \mathrm{UIC}}\right)$, bound intracellular compound $\left(C_{\mathrm{HEP}, \mathrm{BIC}}\right)$, and intracellular binding sites available for nonspecific binding $\left(C_{\mathrm{HEP}, \mathrm{AST}}\right)$.

$$
\begin{aligned}
& V_{\mathrm{HEP}, \mathrm{EC}} \cdot \frac{d C_{\mathrm{HEP}, \mathrm{UEC}}}{d t}=\mathrm{CL}_{\mathrm{HEP}, \mathrm{pass}} \cdot\left(C_{\mathrm{HEP}, \mathrm{UIC}}-C_{\mathrm{HEP}, \mathrm{UEC}}\right) \\
& -k_{\mathrm{HEP}, \text { uptake }} \cdot \frac{C_{\mathrm{HEP}, \mathrm{UEC}}}{C_{\mathrm{HEP}, \mathrm{UEC}}+K_{\mathrm{M}, \mathrm{HEP}, \text { uptake }}} \\
& +k_{\mathrm{HEP}, \text { efflux }} \cdot \frac{C_{\mathrm{HEP}, \mathrm{UIC}}}{C_{\mathrm{HEP}, \text { UIC }}+K_{\mathrm{M}, \mathrm{HEP}, \text { efflux }}} \\
& V_{\mathrm{HEP}, \mathrm{IC}} \cdot \frac{d C_{\mathrm{HEP}, \mathrm{UIC}}}{d t}=-\mathrm{CL}_{\mathrm{HEP}, \text { pass }} \cdot\left(C_{H \mathrm{EP}, \mathrm{UIC}}-C_{\mathrm{HEP}, \mathrm{UEC}}\right) \\
& +k_{\mathrm{HEP}, \text { uptake }} \cdot \frac{C_{\mathrm{HEP}, \mathrm{UEC}}}{C_{\mathrm{HEP}, \mathrm{UEC}}+K_{\mathrm{M}, \mathrm{HEP}, \text { uptake }}} \\
& -k_{\mathrm{HEP}, \text { efflux }} \cdot \frac{C_{\mathrm{HEP}, \mathrm{UIC}}}{C_{\mathrm{HEP}, \mathrm{UIC}}+K_{\mathrm{M}, \mathrm{HEP}, \text { efflux }}} \\
& -k_{\mathrm{HEP}, \text { metabolism }} \cdot \frac{C_{\mathrm{HEP}, \mathrm{UIC}}}{C_{\mathrm{HEP}, \mathrm{UIC}}+K_{\mathrm{M}, \mathrm{HEP}, \text { metabolism }}} \\
& +V_{\mathrm{HEP}, \mathrm{IC}} \cdot k_{\mathrm{off}, \mathrm{HEP}, \mathrm{IC}} \cdot C_{\mathrm{HEP}, \mathrm{BIC}} \\
& -V_{\mathrm{HEP}, \mathrm{IC}} \cdot k_{\mathrm{on}, \mathrm{HEP}, \mathrm{IC}} \cdot C_{\mathrm{HEP}, \mathrm{UIC}} \cdot C_{\mathrm{HEP}, \mathrm{AST}} \\
& \frac{d C_{\mathrm{HEP}, \mathrm{AST}}}{d t}=k_{\mathrm{off}, \mathrm{HEP}, \mathrm{IC}} \cdot C_{\mathrm{HEP}, \mathrm{BIC}}-k_{\mathrm{on}, \mathrm{HEP}, \mathrm{IC}} \cdot C_{\mathrm{HEP}, \mathrm{UIC}} \cdot C_{\mathrm{HEP}, \mathrm{AST}} \\
& \frac{d C_{\mathrm{HEP}, \mathrm{BIC}}}{d t}=-k_{\mathrm{Off}, \mathrm{HEP}, \mathrm{IC}} \cdot C_{\mathrm{HEP}, \mathrm{BIC}}+k_{\mathrm{on}, \mathrm{HEP}, \mathrm{IC}} \cdot C_{\mathrm{HEP}, \mathrm{UIC}} \cdot C_{\mathrm{HEP}, \mathrm{AST}}
\end{aligned}
$$

$V_{\text {HEP,EC }}$ and $V_{\text {HEP,IC }}$ represent the volume of extracellular buffer $(0.5 \mathrm{ml} /$ well $)$ and hepatocytes. $V_{\mathrm{HEP}, \mathrm{IC}}$ is calculated as the product of cell volume per million ( $0.0026 \mathrm{ml}$, in-house value), the number of cells per cell weight ( $2.5 \mathrm{million} / \mathrm{mg}$, in-house value), and the cell weight $\left(A_{\mathrm{HEP}}\right)$ determined in each study. $\mathrm{CL}_{\mathrm{HEP}, \text { pass, }}$ $k_{\mathrm{HEP} \text {,uptake }}, k_{\mathrm{HEP}, \text { efflux }}$, and $k_{\mathrm{HEP} \text {,metabolism }}$ represent passive diffusion clearance, active uptake, active basal efflux, and metabolic rates, respectively. $K_{\mathrm{M}, \mathrm{HEP} \text {,uptake, }}$ $K_{\mathrm{M}, \mathrm{HEP}, \text { efflux }}$, and $K_{\mathrm{M}, \mathrm{HEP} \text {,meta }}$ represent Michaelis-Menten constants for uptake, efflux, and metabolism, respectively. $K_{\mathrm{M}, \mathrm{HEP} \text {,meta }}$ is fixed at values determined from the recombinant P450 assay (Shen et al., 2009) and monkey hepatocyte

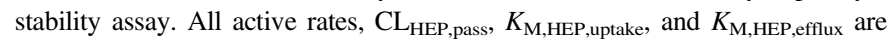
estimated by fitting observed experimental data. In the presence of $1 \mathrm{mM}$ inhibitor $\mathrm{RSV}$, all active processes are fixed at zero based on a previous study (Bi et al., 2017). Although RSV is usually used as a transporter inhibitor, in-house data show that at $1 \mathrm{mM}, \mathrm{P} 450$-mediated metabolism is inhibited as well. In the SCHH study, we could not detect any accumulation in the bile pocket, consistent with the clinical observation that minimal unmetabolized bosentan is identified in the feces after intravenous dosing (Weber et al., 1999); hence, the biliary excretion is fixed at zero. $k_{\text {on,HEP,IC }}$ and $k_{\text {off,HEP,IC }}$ represent the on and off rates in intracellular nonspecific binding. Given the data we have, $k_{\mathrm{On}, \mathrm{HEP}, \mathrm{IC}}$ and $k_{\text {off,HEP,IC }}$ cannot be uniquely identified. As such, the $k_{\text {on,HEP,IC }}$ value is fixed at a value based on the diffusion (Alberty and Hammes, 1958).

The free fraction of extracellular compound $\left(f_{\mathrm{u}, \mathrm{HEP}, \mathrm{EC}}\right)$ is calculated as follows (eq. 5):

$$
f_{\mathrm{u}, \mathrm{HEP}, \mathrm{EC}}=\frac{1}{1+K_{\mathrm{a}, \mathrm{EC}} \cdot A_{\mathrm{HEP}} / V_{\mathrm{HEP}, \mathrm{EC}}}
$$

$K_{\mathrm{a}, \mathrm{EC}}$ is the constant for extracellular nonspecific binding. The amount of compound immobilized on the cell surface or plastic plate is assumed as the product of the total amount of extracellular compound and $\left(1-f_{\mathrm{u}, \mathrm{HEP}, \mathrm{EC}}\right)$. We assume that the immobilized compound not available for transport will be mixed with the intracellular compound during cell lysis. In data fitting, the observed compound accumulation in the cell is represented as the sum of bound and unbound intracellular compound and the immobilized extracellular compound. Values of fixed parameters are listed in Table 1, whereas values of other parameters are estimated by simultaneously fitting data generated with different dosing concentrations under different assay conditions (i.e., with and without transporter inhibitor and calcium).

All of the mathematical models presented in this article were implemented in

\begin{tabular}{|c|c|c|c|}
\hline Parameter & SCHH & PMH & PRH \\
\hline $\mathrm{CL}_{\text {HEP pass }}(\mu 1 / \mathrm{min}$ per $\mathrm{mg})$ & $10.8(9.7,12)$ & $3.55(2.2,5.3)$ & 13.5 \\
\hline$k_{\text {HEP,uptake }}(\mathrm{pmol} / \mathrm{min}$ per $\mathrm{mg})$ & $96.3(67,140)$ & $24.6(15,39)$ & 488 \\
\hline$K_{\text {M.HEP.uptake }}(\mathrm{nM})$ & $4340(2800,6800)$ & $1280(800,2000)$ & 6400 \\
\hline$k_{\text {HEP efflux }}(\mathrm{pmol} / \mathrm{min}$ per $\mathrm{mg})$ & $0.117(0.084,0.15)$ & $68.5(25,170)$ & \\
\hline$K_{\mathrm{M}, \mathrm{HEP}, \text { efflux }}(\mathrm{nM})$ & 0.0100 & $7100\left(2400,2.1 \times 10^{4}\right)$ & \\
\hline$k_{\text {HEP,metabolism }}(\mathrm{pmol} / \mathrm{min}$ per $\mathrm{mg})$ & $3.08 \times 10^{-8}$ & $5.90 \times 10^{-9}$ & \\
\hline$K_{\mathrm{M}, \mathrm{HEP}, \text { metabolism }}(\mathrm{nM})$ & $6.9 \times 10^{4}$ (fixed) & $2.85 \times 10^{4}$ (fixed) & \\
\hline$k_{\text {On,HEP,IC }}(1 / \mathrm{nM} / \mathrm{min})$ & 60 (fixed) & 60 (fixed) & \\
\hline$k_{\text {off.HEP.IC }}\left(\min ^{-1}\right)$ & $8.45 \times 10^{5}\left(5.8 \times 10^{5}, 1.2 \times 10^{6}\right)$ & $8.45 \times 10^{5}$ (fixed) & \\
\hline Total intracellular binding site (nM) & $3.82 \times 10^{5}\left(3.1 \times 10^{5}, 4.7 \times 10^{5}\right)$ & $3.82 \times 10^{5}$ (fixed) & \\
\hline$K_{\mathrm{a}, \mathrm{EC}}(\mathrm{ml} / \mathrm{mg})$ & $2.75 \times 10^{-3}\left(1.8 \times 10^{-3}, 3.7 \times 10^{-3}\right)$ & $0.0163(0.0133,0.0194)$ & \\
\hline Cell number (million cells/mg measured protein) & 2.5 (fixed) & 2.5 (fixed) & \\
\hline Cell volume $(\mathrm{ml} / \mathrm{million}$ cells $)$ & $2.6 \times 10^{-3}$ (fixed) & $2.6 \times 10^{-3}$ (fixed) & \\
\hline$V_{\text {HEP,EC }}(\mathrm{ml} /$ well $)$ & 0.5 (fixed) & 0.5 (fixed) & \\
\hline
\end{tabular}
MATLAB 2016a (MathWorks, Natick, MA). The parameter optimization and uncertainty analysis were performed with differential evolution and Markov chain Monte Carlo (MCMC) approaches with details provided in part 1 of this work ( $\mathrm{Li}$ et al., 2018).

\section{Monkey and Rat PK and Terminal Studies}

Monkey PK studies (intravenous bolus) were conducted at doses of $0.1,0.33$, $1,3.33,10$, and $20 \mathrm{mg} / \mathrm{kg}$. Monkey terminal measurements were performed at

TABLE 1

Medians and 95\% confidence intervals of estimated parameters in an SCHH model and a PMH model and published parameter values in a PRH model

PRH data are published in a previous study (Ménochet et al., 2012). Medians and confidence intervals are not provided for the parameters with high uncertainty (i.e., the range of the approximated confidence interval is greater than 20 magnitude); the globally optimized value is provided instead. 
TABLE 2

Values of fixed parameters in monkey and rat PBPK models

\begin{tabular}{|c|c|c|c|c|}
\hline Parameter & Monkey & Source & Rat & Source \\
\hline $\mathrm{p} K \mathrm{a}$ & 5.2 (acidic) & In house & 5.2 (acidic) & In house \\
\hline $\log \mathrm{D}_{7.4}$ & 1.3 & In house & 1.3 & In house \\
\hline MW $(\mathrm{g} / \mathrm{mol})$ & 551.6 & In house & 551.6 & In house \\
\hline$K_{\mathrm{M}, \text { liver,uptake }}(\mathrm{nM})$ & 1280 & PMH & 6400 & PRH (Ménochet et al., 2012) \\
\hline$K_{\mathrm{M}, \text { liver,metabolism }}(\mathrm{nM})$ & $2.85 \times 10^{4}$ & Suspended monkey hepatocyte & & \\
\hline$k_{\text {on }}(1 / \mathrm{nM} / \mathrm{h})$ & 3600 & Alberty and Hammes (1958) & 3600 & Alberty and Hammes (1958) \\
\hline$k_{\text {off,plasma }}\left(\mathrm{h}^{-1}\right)$ & $2.41 \times 10^{8}$ & Supplemental Material & $5.88 \times 10^{7}$ & Supplemental Material \\
\hline$k_{\mathrm{off}, \mathrm{RBC}}\left(\mathrm{h}^{-1}\right)$ & $7.29 \times 10^{5}$ & Supplemental Material & $5.00 \times 10^{6}$ & Supplemental Material \\
\hline$k_{\text {off,liver,tissue }}\left(\mathrm{h}^{-1}\right)$ & $4.80 \times 10^{7}$ & SCHH & $4.80 \times 10^{7}$ & SCHH \\
\hline Binding site in plasma (nM) & $1.47 \times 10^{6}$ & Supplemental Material & $1.02 \times 10^{6}$ & Supplemental Material \\
\hline Binding site in RBCs (nM) & $1.10 \times 10^{4}$ & Supplemental Material & $3.35 \times 10^{4}$ & Supplemental Material \\
\hline Binding site liver tissue (nM) & $3.82 \times 10^{5}$ & SCHH & $3.82 \times 10^{5}$ & SCHH \\
\hline $\mathrm{HCT}$ & 0.431 & In house & 0.540 & In house \\
\hline $\mathrm{CL}_{\text {systemic,blood,pass }}(\mathrm{l} / \mathrm{h})$ & 41.7 & As described in Li et al. (2018) & 3.94 & As described in Li et al. (2018) \\
\hline $\mathrm{CL}_{\text {liver,blood,pass }}(\mathrm{l} / \mathrm{h})$ & 3.89 & As described in Li et al. (2018) & 0.591 & As described in $\mathrm{Li}$ et al. (2018) \\
\hline
\end{tabular}

HCT, hematocrit; MW, molecular weight; RBC, red blood cell.

$10 \mathrm{mg} / \mathrm{kg}$. Rat PK studies (intravenous bolus) were conducted at doses of 0.1 , $0.33,1,3.33$, and $10 \mathrm{mg} / \mathrm{kg}$. Rat terminal measurements were performed at $10 \mathrm{mg} / \mathrm{kg}$ and with a $3.5 \mathrm{mg} / \mathrm{kg}$ 4-hour intravenous infusion study. All animal care and in vivo procedures were in accordance with guidelines of the Pfizer Animal Care and Use Committee. Details on methods for animal studies are provided in the Supplemental Material.

The PBPK model structure developed in part 1 of this work (Li et al., 2018) was used to analyze the animal data. Since only single intravenous escalating doses were modeled, the simulations were not sensitive to the absorption and P450 induction, which were excluded from the final model. Values for the physiologic parameters are provided in Supplemental Table 1. The binding kinetics in plasma and red blood cells were estimated using data from in vitro assays (Supplemental Fig. 1) and a model structure described in part 1 of this work (Li et al., 2018). The passive diffusion clearance between plasma and red blood cells was determined as described by Li et al. (2018). The binding kinetics in the liver tissue were assumed to be the same as those in the hepatocyte uptake assays. Michaelis-Menten constants of hepatic active uptake ( $\left.K_{\mathrm{M}, \text { liver,uptake }}\right)$ for monkeys and rats were fixed at values determined from $\mathrm{PMH}$ and plated rat hepatocyte (PRH) studies. The Michaelis-Menten constant of hepatic metabolism $\left(K_{\mathrm{M}, \text { liver,metabolism }}\right)$ for monkeys was fixed at a value determined from the monkey hepatocyte stability assay, whereas this parameter was determined for rats by fitting in vivo data because in vitro data were not available. Parameters with fixed values are listed in Table 2. For both species, the Michaelis-Menten constant of hepatic active basal efflux $\left(K_{\mathrm{M} \text {,liver,efflux }}\right)$, rates for uptake, metabolism, and basal efflux ( $k_{\text {liver,uptake, }}$, $k_{\text {liver,metabolism }}$, and $k_{\text {liver,efflux }}$ ), hepatic passive diffusion clearance $\left(\mathrm{CL}_{\text {liver,pass }}\right)$, the concentration of endothelin receptors (ETs), the ET $k_{\text {off }}$ rate, and a scaler for the tissue-to-unbound plasma partition coefficient $\left(K \mathrm{p}_{\mathrm{u}}\right)$ were determined by fitting in vivo plasma and liver data from various dosing groups simultaneously.

\section{Predicting Human Systemic and Liver PK with In Vitro Data, Animal Data, and Mechanistic Models}

For both methods described below, the PBPK model structure and values of fixed parameters for humans (including binding parameters and $K_{\mathrm{M}}$ estimated from in vitro values) are provided in part 1 of this work (Li et al., 2018), whereas values for $\mathrm{CL}_{\text {liver,pass }}, k_{\text {liver,uptake, }}$, and $k_{\text {liver,metabolism were predicted based on either }}$ in vitro (method 1) or animal data (method 2). The observed systemic exposure after intravenous dosing (Weber et al., 1996, 1999) and deduced liver exposure in part 1 of this work ( $\mathrm{Li}$ et al., 2018) were used to validate predictions.

Method 1: Prediction Using Human Hepatocyte Data. Values for $\mathrm{CL}_{\text {liverpass, }}$

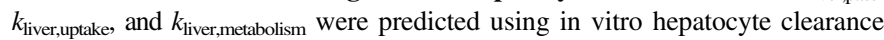
(or rate), physiologic IVIVE scaling factors, and empirical IVIVE scaling factors. The physiologic IVIVE scaling factor is the number of hepatocytes in the liver. The empirical IVIVE scaling factor is not available for the current model in a prospective prediction. Hence, they are approximated with the published values (Li et al., 2014b). The published binding values in both the plasma and liver are generally lower than values used in this study (e.g., published bosentan plasma free fraction $=0.0053$ versus 0.02 in this study and published intracellular free fraction $=0.018$ versus around 0.036 in this study). As such, to estimate "ballpark" values in our predictions, the uptake empirical scaling factor was scaled down by 4 -fold (from 41 to 10.3), whereas the metabolism and the passive diffusion empirical scaling factors were scaled down by a factor of 2 (i.e.,

TABLE 3

Means and 95\% confidence intervals of parameters in human, monkey, and rat PBPK models estimated by fitting in vivo data, and retrospective ratios among different species

\begin{tabular}{|c|c|c|c|c|c|}
\hline Parameter & Human & Monkey & Rat & $\begin{array}{l}\text { Monkey to } \\
\text { Human Ratio }\end{array}$ & $\begin{array}{c}\text { Rat to } \\
\text { Human Ratio }\end{array}$ \\
\hline ET $k_{\text {off }}\left(\mathrm{h}^{-1}\right)$ & $1.61 \times 10^{4}\left(7200,3.4 \times 10^{4}\right)$ & $1039\left(240,1.1 \times 10^{4}\right)$ & $47.9(4.78,505)$ & 0.0645 & $2.98 \times 10^{-3}$ \\
\hline ET total concentration (nM) & $2750(1400,4900)$ & $2310(1400,4100)$ & $2251(968,3890)$ & 0.840 & 0.819 \\
\hline $\mathrm{CL}_{\text {liver,pass }}(\mathrm{l} / \mathrm{h})$ & $23.9(13,44)$ & $4.86(2.8,8.5)$ & $0.0322\left(8.98 \times 10^{-4}, 0.619\right)$ & 0.203 & $1.35 \times 10^{-3}$ \\
\hline$k_{\text {liver,uptake }}(\mathrm{nmol} / \mathrm{h})$ & $4.78 \times 10^{6}\left(3.8 \times 10^{6}, 6.5 \times 10^{6}\right)$ & $6.25 \times 10^{5}\left(4.6 \times 10^{5}, 9.0 \times 10^{5}\right)$ & $1.12 \times 10^{5}\left(8.9 \times 10^{4}, 3.4 \times 10^{5}\right)$ & 0.131 & 0.0234 \\
\hline$k_{\text {liver,metabolism }}(\mathrm{nmol} / \mathrm{h})$ & $1.00 \times 10^{6}\left(8.0 \times 10^{5}, 1.3 \times 10^{6}\right)$ & $1.67 \times 10^{5}\left(1.5 \times 10^{5}, 1.8 \times 10^{5}\right)$ & $1.50 \times 10^{3}$ & 0.167 & \\
\hline$k_{\text {liver,efflux }}(\mathrm{nmol} / \mathrm{h})$ & $6.00 \times 10^{4}$ & 0.539 & 5.55 & & \\
\hline$K_{\text {m.liver,efflux }}(\mathrm{nM})$ & $1.48 \times 10^{6}$ & $3.5 \times 10^{4}$ & 93.9 & & \\
\hline Nonliver tissue $K \mathrm{p}_{\mathrm{u}}$ scaler & 1 (fixed) & $1.47(0.86,2.3)$ & $4.53(<7.11)$ & 1.47 & 4.53 \\
\hline Body weight $(\mathrm{kg})$ & 70.0 (fixed) & 6.20 (fixed) & 0.250 (fixed) & 0.0886 & $3.57 \times 10^{-3}$ \\
\hline Liver tissue weight $(\mathrm{kg})$ & 1.69 (fixed) & 0.167 (fixed) & 0.0103 (fixed) & 0.0988 & $6.09 \times 10^{-3}$ \\
\hline
\end{tabular}

Because $K_{\mathrm{m}, \text { liver,metabolism }}$ for rats is not available from in vitro studies, it is estimated by fitting in vivo PK data with other parameters. The mean is estimated to be $6430 \mathrm{nM}$ but with a high uncertainty.

Medians and confidence intervals are not provided for the parameters with high uncertainty (i.e., the range of the approximated confidence interval is greater than 20 magnitude). The globally optimized value is provided instead. Values for humans were generated in part 1 of this work (Li et al., 2018); whereas values for rats and monkeys are estimated in this study. 
from 0.31 to 0.155 and from 0.29 to 0.145 ). As a result, $\mathrm{CL}_{\text {liverpass }}$ was predicted to be $10.8 \mu \mathrm{l} / \mathrm{min}$ per $\mathrm{mg}$ protein $\times 1 \mathrm{mg}$ protein/million hepatocytes $\times$ 120 million hepatocytes/gram liver tissue $\times 1.69 \mathrm{~kg}$ human liver tissue $\times$ empirical scaling of $0.145=3.17 \times 10^{5} \mu \mathrm{l} / \mathrm{min}=19.1 \mathrm{l} / \mathrm{h}$. It is worth noting that although $1 \mathrm{mg}$ protein was assumed to be 2.5 million hepatocytes in $\mathrm{SCHH}$ modeling (based on current in-house data) as described above, since published empirical scaling factors are used here, $1 \mathrm{mg}$ protein was assumed to be 1 million hepatocytes for consistency with the published work (Li et al., 2014b). Essentially, the factor of 2.5 million hepatocytes/milligram protein is built into the published empirical scaling factors during data fitting. Similarly, values of $k_{\text {liver,uptake }}$ and $k_{\text {liver,metabolism }}$ were predicted to be $1.20 \times 10^{7}$ and $4.42 \times 10^{5}$ $\mathrm{nmol} / \mathrm{h}$. The in vitro assay cannot provide values for hepatic basal efflux or ET binding; hence, they were both ignored from the prediction. Similarly, the $K \mathrm{p}$ scaler was assumed to be 1 .

Method 2: Prediction Using In Vivo Monkey PK Data. The human values for $\mathrm{CL}_{\text {liver,pass }}, k_{\text {liver,uptake, and }} k_{\text {liver,metabolism were predicted using monkey values }}$ (Table 3) scaled by the liver tissue weight difference $(0.167$ and $1.69 \mathrm{~kg}$ for monkey and human liver tissue, respectively). For example, human $\mathrm{CL}_{\text {liver,pass }}$ was predicted to be $4.861 / \mathrm{h} \times(1.69 / 0.167 \mathrm{~kg})^{\alpha}=49.21 / \mathrm{h}$, where $\alpha$ is an allometry exponent. To our knowledge, no similar prediction for a transporter substrate has been published previously; hence, $\alpha$ is arbitrarily fixed at 1 . Similarly, $k_{\text {liver,uptake }}$ and $k_{\text {liver,metabolism }}$ were predicted to be $6.32 \times 10^{6}$ and $1.69 \times 10^{6} \mathrm{nmol} / \mathrm{h}$. The hepatic basal efflux process cannot be confidently estimated from monkey in vivo data; as such, this process is assumed to be zero in human prediction. Human $K \mathrm{p}_{\mathrm{u}}$ scaler and ET binding parameters were assumed to be the same as monkey values (Table 3).

\section{Results}

\section{Hepatocyte Uptake Assay and Mechanistic Modeling}

The observations and simulations for SCHH and PMH assays are provided in Figs. 1 and 2. In both SCHH and PMH data analyses, the $k_{\mathrm{HEP}, \text { metabolism }}$ values were minimal. The active efflux transport process cannot
A

(

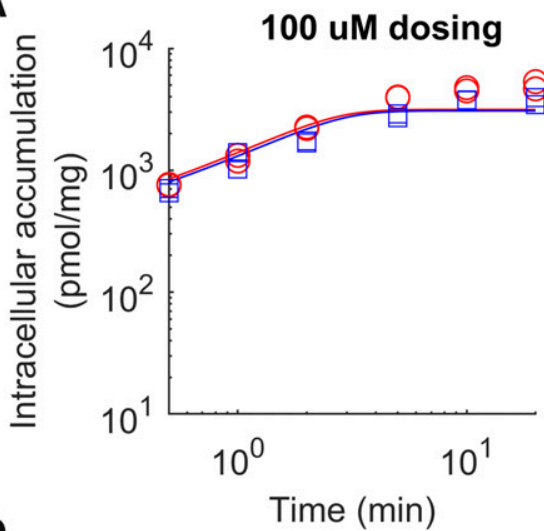

D
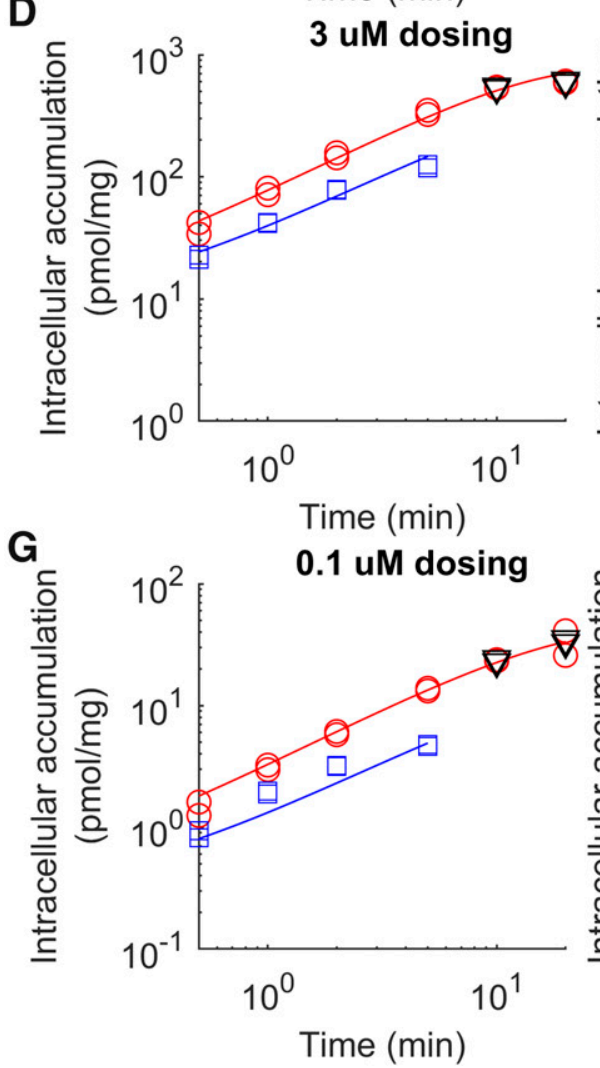

B
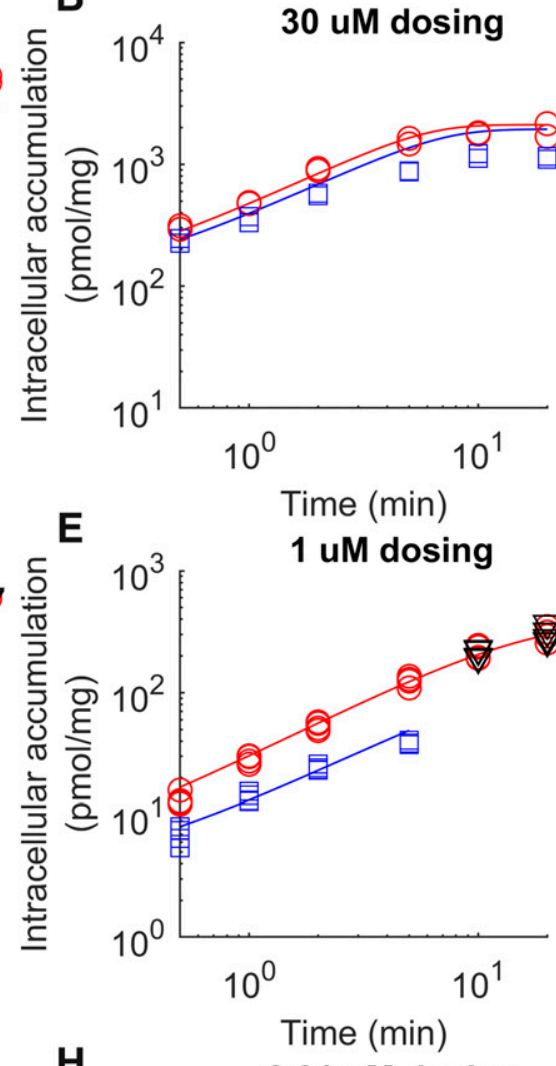
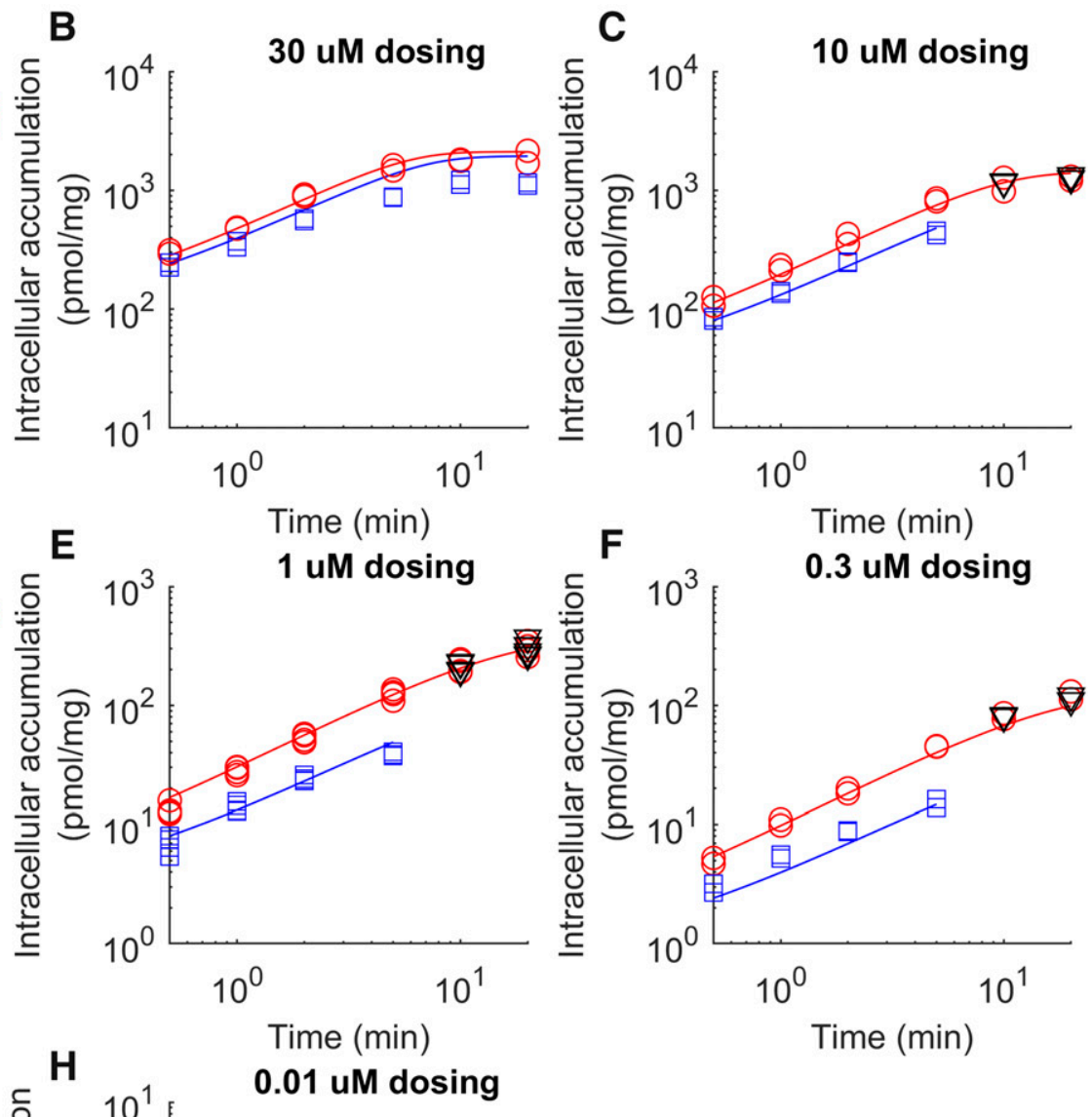

F

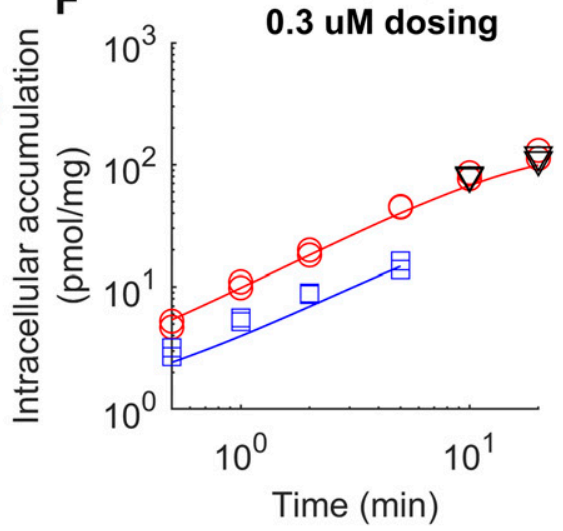

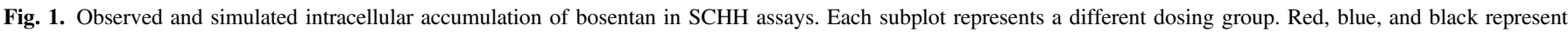

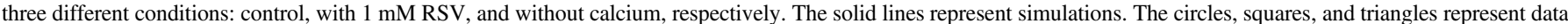
in different conditions: control, with $1 \mathrm{mM} \mathrm{RSV}$, and without calcium, respectively. Both horizontal and vertical axes are in log scale. 

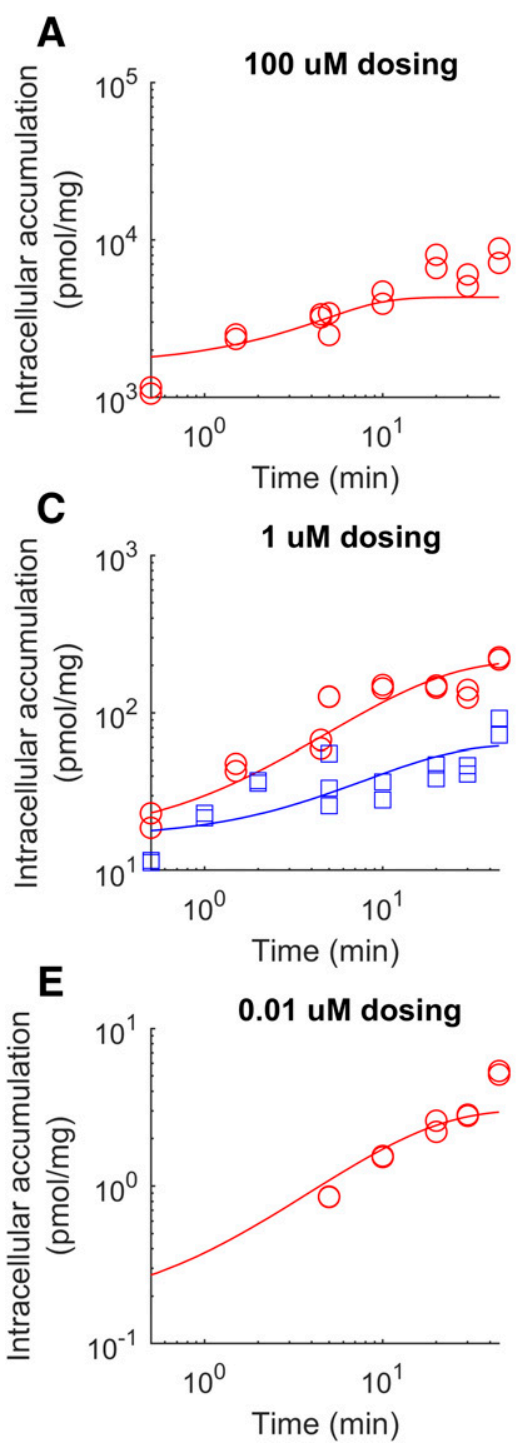

B
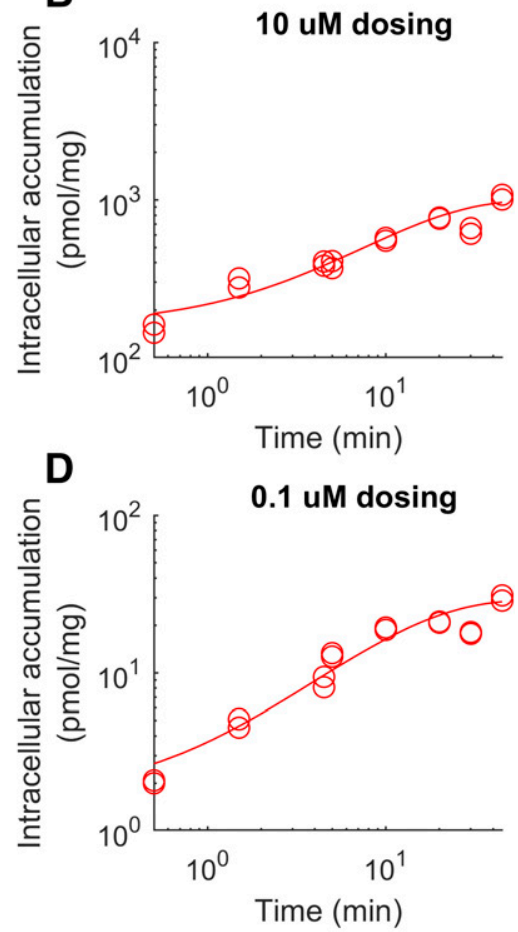

Fig. 2. Observed and simulated intracellular accumulation of bosentan in PMH assays. Each subplot represents a different dosing group. Red and blue represent two different conditions: without and with $1 \mathrm{mM}$ RSV, respectively. The solid lines represent simulations. The circles and squares represent data in different conditions: control and with $1 \mathrm{mM} \mathrm{RSV}$, respectively. Both horizontal and vertical axes are in $\log$ scale. be identified given the current data, due to large uncertainty in estimating $K_{\mathrm{M}, \mathrm{HEP} \text {,efflux }}$. For binding parameters, $\mathrm{PMH}$ data are not sufficient to provide confident estimates; hence, we assumed that PMHs shared the same binding values as SCHHs. All other parameters can be precisely determined (Table 1). For comparison purposes, we also provide the published PRH rates (Ménochet et al., 2012) in Table 1. It is worth noting that although ratios between $k_{\mathrm{HEP} \text {,uptake }}$ and $K_{\mathrm{M}, \mathrm{HEP} \text {,uptake were both around }}$

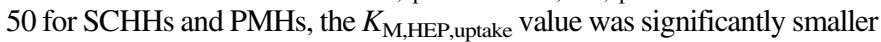
in PMHs. In addition, PMHs also had a small $\mathrm{CL}_{\mathrm{HEP} \text {,pass }}$ value.

\section{Monkey and Rat PK Studies and Mechanistic Modeling}

Our model can reasonably describe monkey (Fig. 3) and rat (Fig. 4) PK data in both plasma and liver samples. All monkey parameters except $k_{\text {liver,efflux }}$ and $K_{\text {m,liver,efflux }}$ could be confidently estimated (Table 3), a similar phenomenon observed in fitting human data as described in part 1 of this work (Li et al., 2018). The monkey pseudo steady-state unbound liver tissue-to-unbound plasma ratio $\left(K \mathrm{p}_{\mathrm{uu}}\right)$ and its $95 \%$ confidence intervals were $51.3(39,70)$ for $0.1 \mathrm{mg} / \mathrm{kg}$ dosing and $66.5(53,83)$ for $20 \mathrm{mg} / \mathrm{kg}$ dosing. Median liver $K \mathrm{p}_{\mathrm{uu}}$ estimates for other dosing groups were between 52.3 and 60.0. In the rat model, parameters for hepatic metabolism and efflux could not be confidently identified (Table 3 ). $K \mathrm{p}_{\mathrm{uu}}$ and its $95 \%$ confidence intervals were 83.4
(16, 130) for $0.1 \mathrm{mg} / \mathrm{kg}$ dosing and $87.0(35,142)$ for $10 \mathrm{mg} / \mathrm{kg}$ dosing.

It is worth noting that if the monkey PK data were analyzed with a noncompartment analysis or a traditional two-compartment model instead of using the mechanistic model, the estimated blood clearance would be close to or greater than the hepatic blood flow in some dosing groups (Supplemental Table 2), because noncompartment or traditional two-compartment analysis ignores nonlinear binding kinetics in blood.

\section{Prospectively Predicting Human Systemic and Liver PK with} In Vitro Data, Animal Data, and Mechanistic Models

Both methods 1 (in vitro data based) and 2 (monkey data based) provide predictions that largely match the clinical PK data after intravenous dosing, but method 2 provides a much more reasonable prediction, particularly in the distribution phase (Fig. 5). Using predicted hepatic processes from hepatocytes, the predicted human liver $K \mathrm{p}_{\mathrm{uu}}$ was 142 for both 10 and $750 \mathrm{mg}$ intravenous dosing (Fig. 5). Alternatively, utilizing monkey in vivo data-predicted hepatic processes, the predicted human $K \mathrm{p}_{\mathrm{uu}}$ values were 21.1 and 22.9 for 10 and $750 \mathrm{mg}$ intravenous dosing, which are much closer to the value (i.e., 34.9) we estimated with human clinical data in part 1 of this work (Li et al., 2018). The ability to estimate ET binding (particularly the in vivo concentration of the 

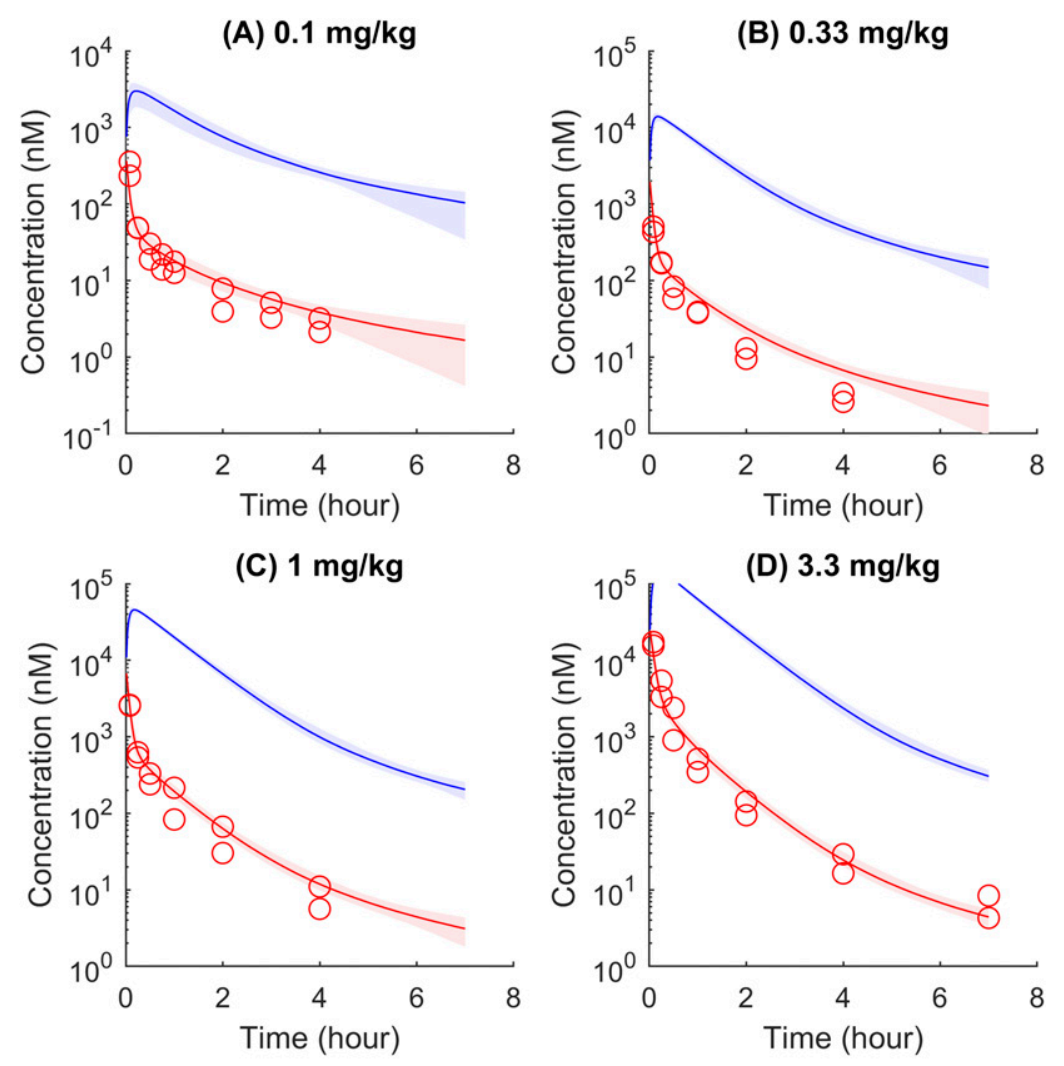

Fig. 3. Observed and simulated monkey PK time courses. Red and blue indicate total plasma and total liver concentrations, respectively. The solid lines and shaded areas represent the median simulations, and $95 \%$ confidence intervals generate with parameter values identified in Markov chain Monte Carlo. The circles and squares represent data from PK and terminal studies, respectively.
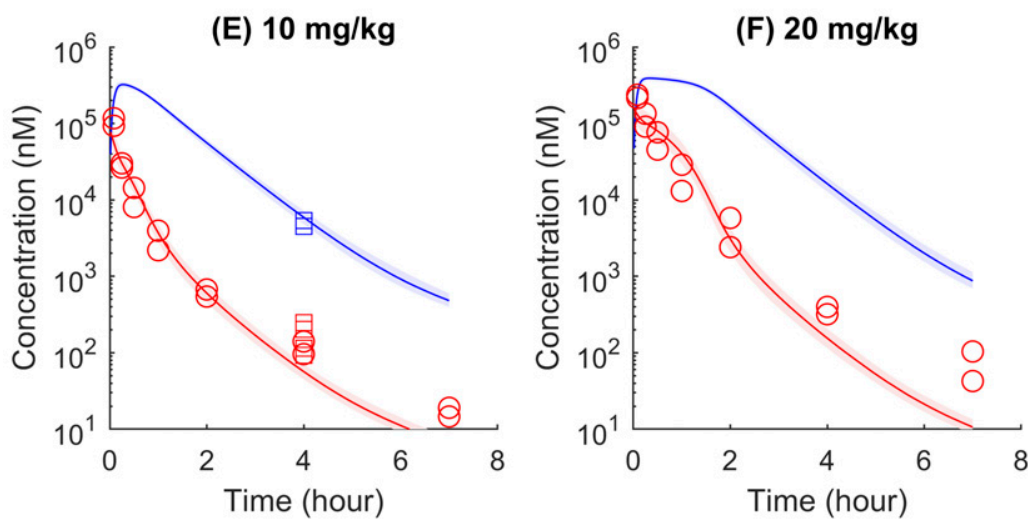

receptor) using method 2 also contributes to a better systemic prediction (elimination phase), although liver predictions are similar with and without modeling ET binding.

\section{Retrospective Analyses on Two Prediction Approaches}

In addition to the prospective predictions described above, retrospective analyses were performed to better understand IVIVE. In method 1, the retrospective empirical scaling factors were back-calculated, as the ratios of hepatic processes values estimated in part 1 of this work ( $\mathrm{Li}$ et al., 2018) using clinical data, to the physiologically scaled values based on hepatocyte data. The prospective empirical scaling factors we used were about 2-fold greater for $k_{\text {liver,uptake }}$ but were 1.5 and 2 times lower for $\mathrm{CL}_{\text {liver,pass }}$ and $k_{\text {liver,metabolism }}$ than the retrospective scaling factors (Table 4). Using prospective scaling factors, the overpredicted uptake leads to an underpredicted systemic concentration in the distribution phase, whereas underpredicted metabolism brings the systemic concentration back within the "normal" range during the elimination phase (Fig. 5). Although the two mispredicted processes may cancel each other's impact on systemic exposure, both will result in overpredicted liver exposure.

For method 1, we also calculated an empirical scaling factor for monkeys and rats in retrospective analyses (Table 4) to better understand whether different species may have similar empirical scaling for the same compound. Monkeys require different scaling factors from humans. Because we cannot confidently estimate in vivo $k_{\text {liver,metabolism }}$ for rats, only the empirical scaling factors for $\mathrm{CL}_{\text {liver,pass }}$ and $k_{\text {liver,uptake }}$ are provided, and we cannot determine whether rats share the same empirical scaling with humans overall.

For method 2, even with the allometry exponent $(\alpha)$ arbitrarily fixed at 1 , the model reasonably predicts systemic and liver exposure. To understand an $\alpha$ value that best describes the data, a retrospective calculation determined this value to be 0.88 . The ratios between values of monkey and human rates were around 0.15 for all three hepatic processes (Table 3), which makes it possible to use the same $\alpha$ across different hepatic processes in consistently translating monkey rates to human rates based on liver weights. Although human rates of hepatic 

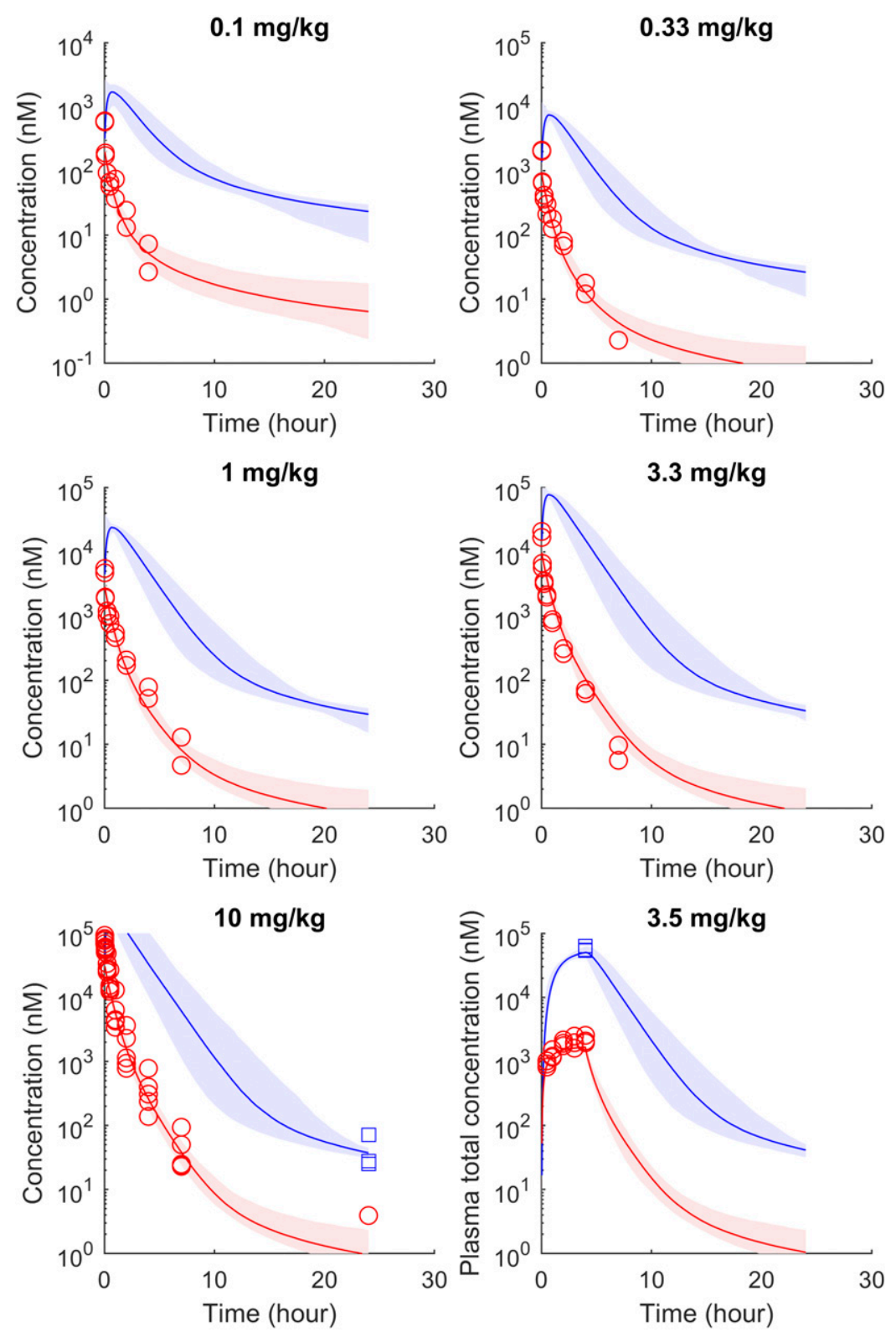

Fig. 4. Observed and simulated rat PK time courses. Red and blue indicate total plasma and total liver concentrations, respectively. The solid lines and shaded areas represent the median simulations, and 95\% confidence intervals generate with parameter values identified in Markov chain Monte Carlo. The circles and squares represent data from PK and terminal studies, respectively. processes were scaled from monkey in vivo values, Michaelis-Menten constants were still fixed at values from human hepatocyte assays. In the absence of in vitro hepatocyte data, these constants estimated from monkey in vivo data cannot accurately predict human PK (Supplemental Fig. 2).

\section{Discussion}

Prospectively predicting PK of hepatic transporter substrates in clinical studies remains a challenging task, particularly in predicting liver exposures. Although several approaches have been developed to face this challenge, most focus only on systemic exposure. In addition, due to the use of compound-dependent empirical scaling factors estimated by fitting clinical observation, these published works are more retrospective analyses but are rarely tested prospectively. Similarly, when predicting liver exposure-driven DDI and toxicity of transporter substrates, most published approaches allow floating parameters to match clinical observations. Hence, confidence in using these approaches in real-world drug discovery and development is low.
There are two obstacles impeding progress: 1) comprehensive knowledge of human transporter activity and 2) human liver exposure data to valid prediction approaches developed. The former problem is a result of the latter. Without direct measurement of the liver, researchers can only use indirect surrogates in most studies (e.g., systemic exposure as a surrogate of metabolism, or PK of a victim compound as a surrogate of DDI). Unfortunately, for most compounds, the relationship between liver exposure and these surrogates is not well understood. Hence, sometimes even a retrospective analysis on clinical observations may not yield a confident result, let alone a prospective prediction.

In this study, we aimed to 1) understand human liver exposure of bosentan, a transporter substrate, and 2) develop and/or validate translational approaches to predict human liver exposure, using currently available preclinical tools. Details on the first goal are provided in part 1 of this work (Li et al., 2018). Briefly, a deduction approach based on physiologic analysis of the observed systemic exposure was used to understand liver exposure in the healthy population. For a compound with hepatic metabolism like bosentan, the deduction approach seems to 

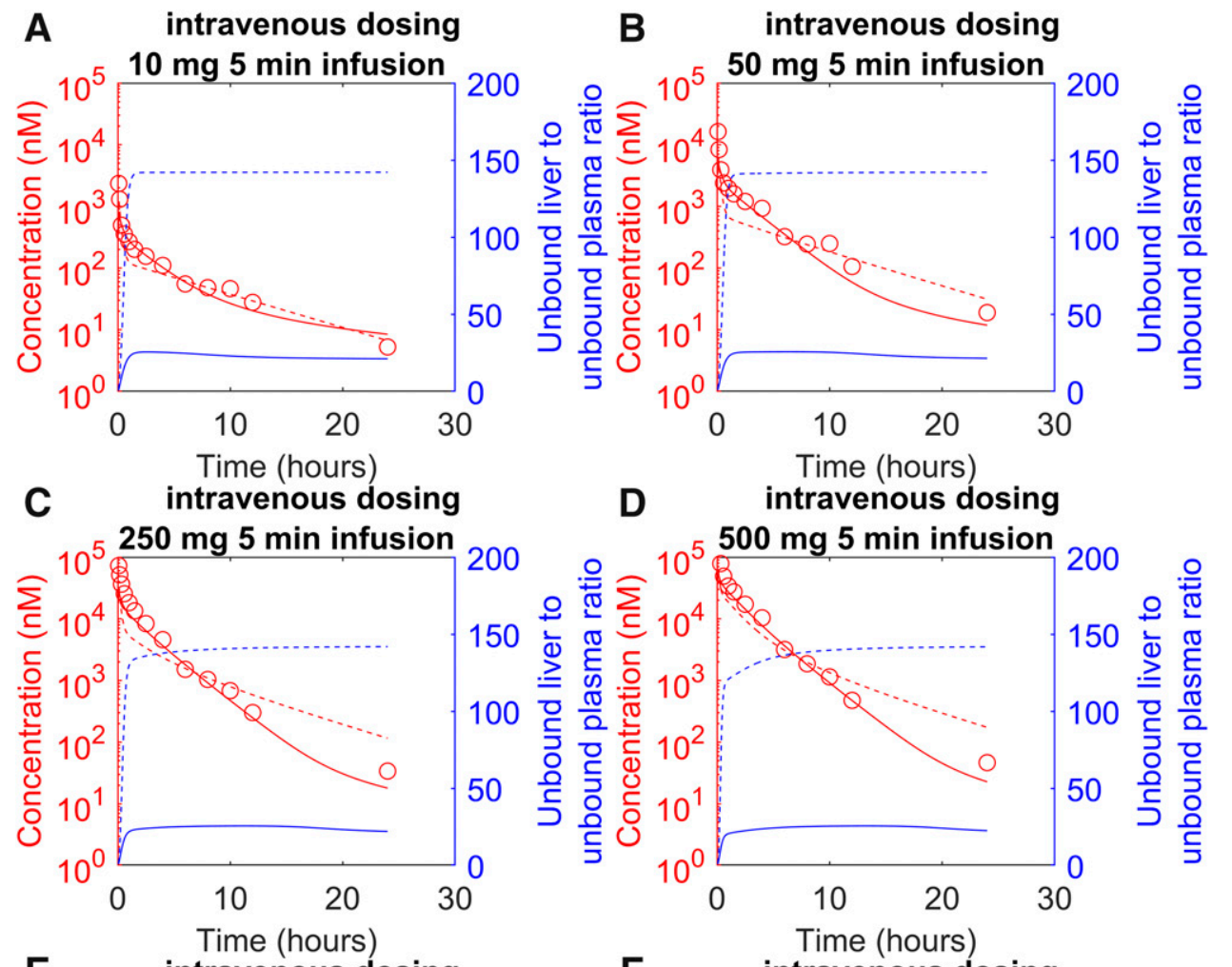

Fig. 5. Prospectively predicted systemic PK of bosentan and its unbound liver-to-unbound plasma ratio. The dashed and solid lines represent predictions based on in vitro data (method 1) and monkey in vivo data (method 2). The circles represent clinical observation. Red and blue represent total systemic concentration (left axis) and unbound liver-to-unbound plasma ratio (right axis).
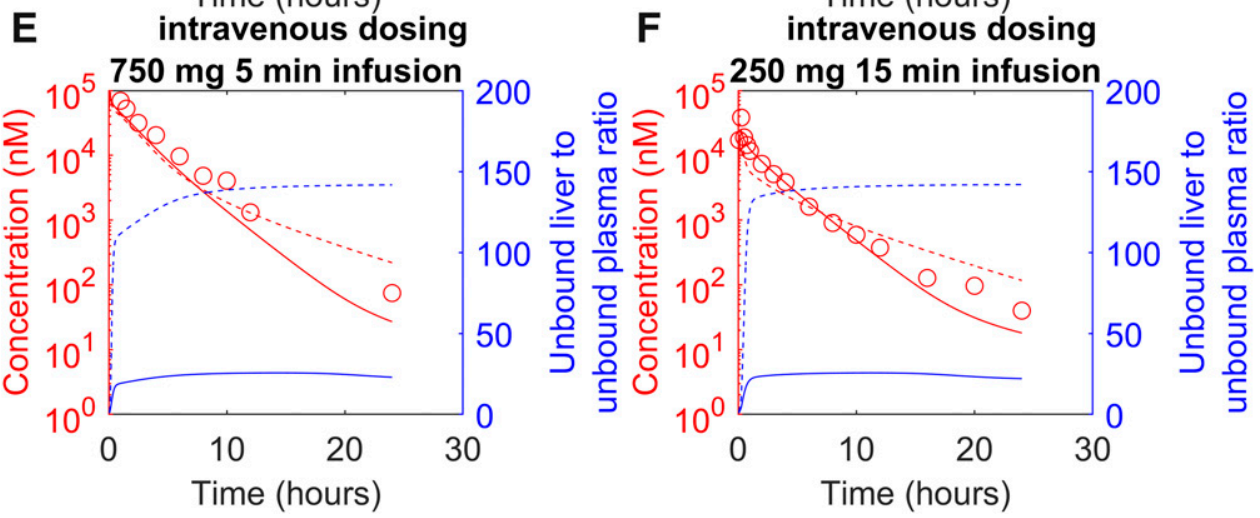

currently be the only viable one to predict liver exposure. The two key criteria for such an approach are accuracy and precision: confidence in accuracy resides in the conservation of mass, while precision is tested with statistical approaches. Unlike most other hepatic transporter substrates, bosentan does not have additional disposition pathways such as biliary excretion and enterohepatic recirculation, simplifying the problem in analyzing mass conservation through PBPK modeling. Although challenges in modeling bosentan PK exist (e.g., potentially nonlinear binding processes), they can still be studied with in vitro assays. In addition, there are sufficient clinical data to generate precise parameter estimates and liver predictions, which make bosentan unique from other compounds that have been studied previously ( $\mathrm{Li}$ et al., 2016). Both advantages help to more confidently establish a relationship among hepatic disposition, exposure, and their systemic surrogate.

Here, several approaches using readily available preclinical tools were tested against the liver exposure deduced in part 1 of this work (Li et al., 2018). Direct liver measurements in terminal animal studies were tested first. Liver exposures of species with great similarity to humans can even be quite different from humans (e.g., $K \mathrm{p}_{\mathrm{uu}}$ of about 60 in the monkey but about 35 in humans for bosentan).
Regarding the mechanistic approaches, although both methods use the same model structure and can describe the systemic exposure, newly proposed method 2 (i.e., hepatic process rates estimated using monkey data) shows a more accurate prediction. As for predicting liver exposure, method 1 (i.e., hepatic process rates estimated using hepatocyte data) leads to a liver $K \mathrm{p}_{\mathrm{uu}}$ of 142 , which is substantially greater than the value (34.9) deduced using clinical data. Conversely, the $K \mathrm{p}_{u u}$ value from method 2 is more consistent with the deduced value. A set of parameter values that can reasonably simulate systemic exposure or clearance cannot necessarily predict liver exposure due to uncertainty associated with parameter values. If parameter values are estimated by fitting systemic data, caution should be taken when these fitted values are used to simulate liver exposure or the effects (e.g. pharmacodynamics, DDI, or toxicity) driven by liver exposure. Details on this topic were addressed in a previous publication ( $\mathrm{Li}$ et al., 2016).

In method 1 , the prospective empirical scaling factors leading to overpredicted hepatic uptake were estimated in a previous study by simultaneously fitting human systemic PK of several OATP substrates (Li et al., 2014b). The approach was developed to reduce uncertainty in estimating scaling factors by fitting data of individual compounds. There 
TABLE 4

Physiologically scaled in vivo hepatic clearance processes based on hepatocyte data, and IVIVE ESF

\begin{tabular}{|c|c|c|c|}
\hline Parameter & Human & Monkey & Rat \\
\hline Physiologically scaled $\mathrm{CL}_{\text {liver,pass }}{ }^{a}(\mathrm{l} / \mathrm{h})$ & 131 & 5.10 & 0.901 \\
\hline Prospective diffusion $\mathrm{ESF}^{b}$ & 0.145 & & \\
\hline Retrospective diffusion $\mathrm{ESF}^{c}$ & 0.182 & 0.953 & 0.0357 \\
\hline Physiologically scaled $k_{\text {liver,uptake }}{ }^{d}(\mathrm{nmol} / \mathrm{h})$ & $1.17 \times 10^{6}$ & $3.53 \times 10^{4}$ & $3.26 \times 10^{4}$ \\
\hline Prospective uptake ESF & 10.3 & & \\
\hline Retrospective uptake ESF & 4.08 & 17.7 & 3.96 \\
\hline Physiologically scaled $k_{\text {liver,metabolism }}(\mathrm{nmol} / \mathrm{h})$ & $2.85 \times 10^{6}$ & $1.15 \times 10^{4}$ & \\
\hline Prospective metabolism ESF & 0.155 & & \\
\hline Retrospective metabolism ESF & 0.351 & 0.146 & \\
\hline
\end{tabular}

Hepatic transport processes are predicted with SCHHs, whereas metabolic rates are predicted with suspended hepatocytes (in vitro metabolic rates: human hepatocytes, $234.6 \mathrm{pmol} / \mathrm{min}$ per million; monkey hepatocytes, $798 \mathrm{pmol} / \mathrm{min}$ per million). ESF, empirical scaling factor.

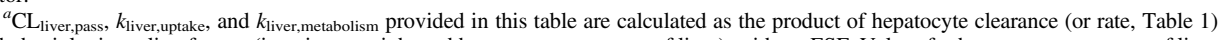
and physiologic scaling factors (i.e., tissue weight and hepatocytes per gram of liver), without ESF. Values for hepatocytes per gram of liver are 120,122 , and 108 million for humans, monkeys, and rats.

${ }^{b}$ Prospective ESF is approximated using published values after accounting for different binding values between publications and this study (see details in the Materials and Methods).

${ }^{c}$ Retrospective ESF is calculated as the ratio of clearance (or rate) estimated by fitting in vivo data (Table 3 ) to the physiologically predicted clearance (or rate) in this table.

${ }^{d}$ For rats, we only provide IVIVE ratio for uptake, due to high uncertainty associated with other parameters in fitting rat in vivo data

are multiple reasons that may lead to a mismatch between the current prediction and the old scaling factors. These scaling factors were developed with a model assuming linear kinetics, while the current model uses nonlinear equations to describe both binding and hepatic processes. In addition, in vitro SCHH assays were performed with different hepatocyte lots in the two studies, which may have different transporter activity. It should not be concluded that method 1 is necessarily worse than method 2 at this time. If the global scaling factor were re-estimated with the current model structure and hepatocyte lots, method 1 might yield improved systemic and liver predictions. In estimating empirical scaling factors by simultaneously fitting clinical data of several compounds, there are also areas that deserve further study. For example, $K \mathrm{p}_{\mathrm{u}}$ values for nonliver tissues are usually fixed at values from in silico approaches (Rodgers and Rowland, 2006). The scaling factor estimation can be confounded if the nonliver $K \mathrm{p}_{\mathrm{u}}$ value is inaccurate for any training compound (Li et al., 2014b,c). We have partly tested another topic: for the same compound, can we estimate empirical scaling factors from animals and apply them to humans (Watanabe et al., 2009)? Based on our retrospective analysis (Table 4), the values of scaling factors for humans and animals can be quite different for compounds like bosentan.

Due to the lack of empirical scaling factors specifically tailored for the current model structure and hepatocyte, we have not fully tested the liver prediction capability of method 1 . From this perspective, method 2 may be a superior approach since it does not require data from other training compounds, although its prediction accuracy should be further validated with additional compounds in the future. Method 2 requires data from both in vivo monkey studies (for hepatic process rates) and in vitro studies (for Michaelis-Menten constants). We have tested prediction without in vitro data, where both rates and Michaelis-Menten constants are estimated using in vivo monkey data. However, the systemic exposure is underpredicted, while liver exposure is overpredicted. A similar phenomenon was published previously with another acidic compound with a molecular weight of 428 (i.e., a potential OATP substrate), GSK269984A (sodium;6-[[5-chloro-2-[(4-chloro-2fluorophenyl)methoxy]phenyl]methyl]pyridine-2-carboxylate). Although rates may be similar between two species, monkeys may have a smaller $K_{\mathrm{M} \text {,liver,uptake }}$ value than humans, which may cause overpredicted uptake. Hence, unless it has been proven that the compounds have large enough $K_{\mathrm{M}}$ values in both monkeys and humans, hepatic processes estimated from monkey in vivo data per se should not be used to predict human PK without accounting for species $K_{\mathrm{M}}$ differences. We also tried to use method 2 but with hepatic rates, $K \mathrm{p}_{\mathrm{u}}$ scalers, and ET binding parameters estimated from rat in vivo data. An allometry exponent of 0.8 seems to best describe the human data; however, the simulation still overpredicts liver exposure but underpredicts systemic exposure (Supplemental Fig. 3).

In conclusion, using bosentan data, we have provided an example for prospectively predicting hepatic exposure of a transporter substrate. The accuracies of the new and previously established approaches have been validated against the human liver $K \mathrm{p}_{\mathrm{uu}}$ deduced in part 1 of this work (Li et al., 2018). The new approach shows the highest accuracy in this study.

\section{Acknowledgments}

We thank Dr. Manthena V. Varma (Pfizer Inc.) for helpful discussions and Karen Atkinson (Pfizer Inc.) for assistance with manuscript editing.

\section{Authorship Contributions}

Participated in research design: Li, Kimoto, Niosi, Tess, Lin, Tremaine, Di. Conducted experiments: Kimoto, Niosi, Lin.

Contributed new reagents or analytic tools: Li.

Performed data analysis: Li, Kimoto, Niosi, Tess, Lin, Tremaine, Di.

Wrote or contributed to the writing of the manuscript: Li, Kimoto, Niosi, Lin, Di.

\section{References}

Alberty RA and Hammes GG (1958) Application of the theory of diffusion-controlled reactions to enzyme kinetics. J Phys Chem 62:154-159.

Bi YA, Scialis RJ, Lazzaro S, Mathialagan S, Kimoto E, Keefer J, Zhang H, Vildhede AM, Costales C, Rodrigues AD, et al. (2017) Reliable rate measurements for active and passive hepatic uptake using plated human hepatocytes. AAPS J 19:787-796.

Li R, Barton HA, and Varma MV (2014a) Prediction of pharmacokinetics and drug-drug interactions when hepatic transporters are involved. Clin Pharmacokinet 53:659-678.

Li R, Barton HA, Yates PD, Ghosh A, Wolford AC, Riccardi KA, and Maurer TS (2014b) A "middle-out" approach to human pharmacokinetic predictions for OATP substrates using physiologically-based pharmacokinetic modeling. J Pharmacokinet Pharmacodyn 41:197-209.

Li R, Ghosh A, Maurer TS, Kimoto E, and Barton HA (2014c) Physiologically based pharmacokinetic prediction of telmisartan in human. Drug Metab Dispos 42:1646-1655.

Li R, Maurer TS, Sweeney K, and Barton HA (2016) Does the systemic plasma profile inform the liver profile? Analysis using a physiologically based pharmacokinetic model and individual compounds. AAPS J 18:746-756.

Li R, Niosi M, Johnson N, Tess DA, Kimoto E, Lin J, Yang X, Riccardi KA, Ryu S, El-Kattan AF, Maurer TS, Tremaine LM, and Di L (2018) A study on pharmacokinetics of bosentan with systems modeling, Part 1: translating systemic plasma concentration to liver exposure in healthy subjects. Drug Metab Dispos 46:346-356.

Ménochet K, Kenworthy KE, Houston JB, and Galetin A (2012) Simultaneous assessment of uptake and metabolism in rat hepatocytes: a comprehensive mechanistic model. $J$ Pharmacol Exp Ther 341:2-15. 
Morse BL, Cai H, MacGuire JG, Fox M, Zhang L, Zhang Y, Gu X, Shen H, Dierks EA, Su H, et al. (2015) Rosuvastatin liver partitioning in cynomolgus monkeys: measurement in vivo and prediction using in vitro monkey hepatocyte uptake. Drug Metab Dispos 43 $1788-1794$

Rodgers T and Rowland M (2006) Physiologically based pharmacokinetic modelling 2: predicting the tissue distribution of acids, very weak bases, neutrals and zwitterions. J Pharm Sci 95: $1238-1257$.

Shen G, Yao M, Fura A, and Zhu M (2009) In vitro metabolite identification and cytochrome $\mathrm{P} 450$ reaction phenotyping of bosentan, a dual endothelin receptor antagonist, in Proceedings of the American Association of Pharmaceutical Scientists Annual Meeting and Exposition; 2009 Nov 9-11; Los Angeles, CA. American Association of Pharmaceutical Scientists, Arlington, VA.

Shimizu K, Takashima T, Yamane T, Sasaki M, Kageyama H, Hashizume Y, Maeda K, Sugiyama Y, Watanabe Y, and Senda M (2012) Whole-body distribution and radiation dosimetry of [11C]telmisartan as a biomarker for hepatic organic anion transporting polypeptide (OATP) 1B3. Nucl Med Biol 39:847-853.
Watanabe T, Kusuhara H, Maeda K, Shitara Y, and Sugiyama Y (2009) Physiologically based pharmacokinetic modeling to predict transporter-mediated clearance and distribution of pravastatin in humans. J Pharmacol Exp Ther 328:652-662.

Weber C, Gasser R, and Hopfgartner G (1999) Absorption, excretion, and metabolism of the endothelin receptor antagonist bosentan in healthy male subjects. Drug Metab Dispos 27: $810-815$.

Weber C, Schmitt R, Birnboeck H, Hopfgartner G, van Marle SP, Peeters PA, Jonkman JH, and Jones CR (1996) Pharmacokinetics and pharmacodynamics of the endothelin-receptor antagonist bosentan in healthy human subjects. Clin Pharmacol Ther 60:124-137.

Address correspondence to: Rui Li, Systems Modeling and Simulation, Medicine Design, Pfizer Worldwide R\&D, 610 Main Street, Cambridge, MA 02139. E-mail: rui. li5@pfizer.com 